\title{
Smartphone-assisted detection of nucleic acids by light-harvesting FRET-based nanoprobe
}

\author{
Caterina Severi, Nina Melnychuk, and Andrey S. Klymchenko*
}

Laboratoire de Biophotonique et Pathologies, Faculté de Pharmacie, UMR 7021 CNRS, Université de Strasbourg, 74, Route du Rhin, 67401 Cedex, Illkirch, France

*Corresponding author. E-mail address: andrey.klymchenko@unistra.fr (A. S. Klymchenko); tel: +33 3688542 55, Fax: +33368 854313 .

\begin{abstract}
Point-of-care assays for optical detection of biomolecular markers attract growing attention, because of their capacity to provide rapid and inexpensive diagnostics of cancer and infectious diseases. Here, we designed a nanoprobe for detection of nucleic acids compatible with a smartphone RGB camera. It is based on light-harvesting polymeric nanoparticles (NPs) encapsulating green fluorescent donor dyes that undergo efficient Förster Resonance Energy Transfer (FRET) to red fluorescent acceptor hybridized at the particle surface. Green-emitting NPs are based on rhodamine 110 and 6G dyes paired with bulky hydrophobic counterions, which prevent dye selfquenching and ensure efficient energy transfer. Their surface is functionalized with a capture DNA sequence for cancer marker survivin, hybridized with a short oligonucleotide bearing FRET acceptor ATTO647N. Obtained 40nm poly(methyl methacrylate)-based NP probe, encapsulating octadecyl rhodamine 6G dyes with tetrakis(perfluoro-tert-butoxy)aluminate counterions ( 6000 dyes per NP), and bearing 65 acceptors, shows efficient FRET with $>20 \%$ quantum yield and a signal amplification (antenna effect) of 25. It exhibits ratiometric response to the target DNA by FRET acceptor displacement and enables DNA detection in solution by fluorescence spectroscopy (limit of detection $3 \mathrm{pM}$ ) and on surfaces at the single-particle level using two-color fluorescence microscopy. Using a smartphone RGB camera, the nanoprobe response can be readily detected at 10 pM target in true color and in red-to-green ratio images. Thus, our FRET-based nanoparticle biosensor enables detection of nucleic acid targets using a smartphone coupled to an appropriate optical setup, opening the way to simple and inexpensive point-of-care assays.
\end{abstract}

Keywords: Smartphone-based detection; Detection of nucleic acids; Fluorescence microscopy; Polymeric nanoparticles; Nanoantenna; Point-of-care diagnostics 
Graphical abstract:

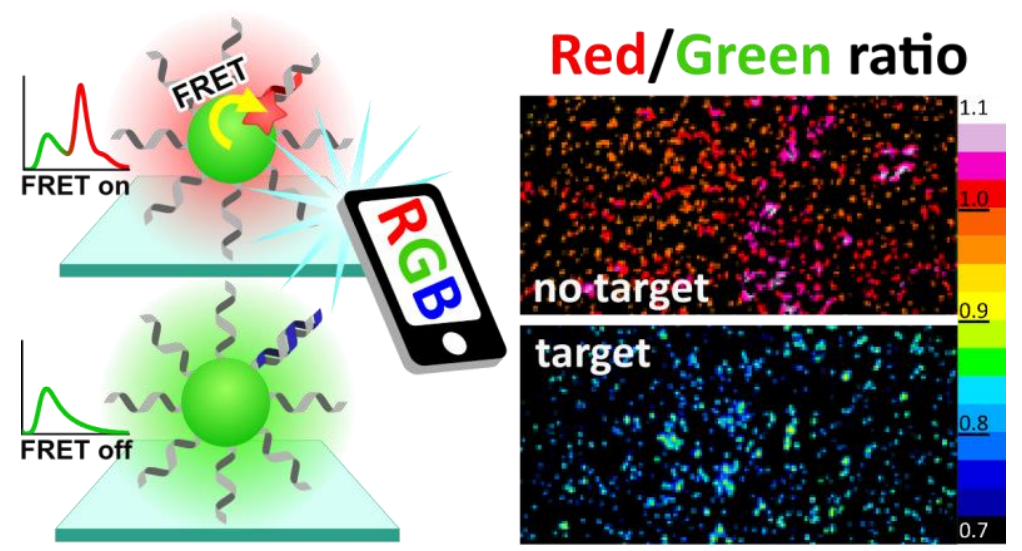




\section{Introduction}

Point-of-care (PoC) diagnostics is a forefront research direction devoted to greatly accelerate early diagnostics of diseases (Yang et al., 2019). Ideally, point-of-care testing devices should be inexpensive, portable and easy to use while still being sensitive and selective towards the target species. A great deal of efforts has been devoted to the construction of the electrochemical biosensors, which match these requirements (Chen and Chatterjee, 2013; Dakshayini et al., 2019; Roy et al. 2019; Shetti et al., 2019). Smartphones are exceptional candidates in the biosensor development due to their almost universal prevalence (Zhang and Liu, 2016). Fluorescence smartphonebased detection systems were developed for a variety of scopes, such as: blood analysis (Breslauer et al., 2009), detection of viruses (Wei et al., 2013), bacteria (Zhu et al., 2012), cells (Breslauer et al., 2009), proteins (Coskun et al., 2013), nucleic acids (Wei et al., 2014; Xu et al., 2020) and DNA sequencing (Kühnemund et al., 2017). However, smartphone cameras are generally less sensitive than the devices commonly used in research, featuring sensitivity down to single-molecule detection (Kanchi et al., 2018). Sensitivity is a vital quality of point-of-care devices, in order to permit the detection of very low concentration of analyte, thus allowing early diagnosis of diseases.

The inherent limitation of fluorescence-based assays is the limited brightness of fluorescent dyes (Grimm et al., 2015). This has led to the development of many amplification strategies for which single molecular recognition event triggers a response equivalent to hundreds of fluorescent dyes (Scrimin and Prins, 2011). This amplification can be achieved by increasing the number of target molecules, as in polymerase chain reaction (PCR) and methods based on enzymatic amplification or hybridization chain reactions (Ali et al., 2014; Choi et al., 2010). An alternative approach is direct amplification of the fluorescence signal from the dye, which is attractive for PoC applications owing to its simplicity. In this respect, three systems have already been established: (i) plasmonic nanostructures, which amplify the fluorescence of single dyes located at a precisely controlled distance (Acuna et al., 2012; Wei et al., 2017); (ii) light-harvesting with conjugated polymers (Jiang and McNeill, 2017), in which a large number of $\pi$ conjugated aromatic units efficiently transfer the excitation energy to one energy acceptor (Rochat and Swager, 2013) and (iii) light-harvesting with dye-loaded fluorescent polymeric nanoparticles (Trofymchuk et al., 2017).

Previously, we developed dye-loaded polymeric NPs encapsulating hundreds of rhodamine dyes paired with a bulky hydrophobic counterion (Reisch et al., 2014; Reisch and Klymchenko, 2016). The use of the bulky counterion ensures efficient dye encapsulation without leakage in biological media, prevents aggregation-caused quenching of dyes at high loading and at the same time assembles dyes inside the polymeric matrix with relatively short spacing, inducing a collective behavior of dyes inside the nanoparticle (Andreiuk et al., 2019; Reisch et al., 2017). This collective behavior was exploited to design a giant light-harvesting nanoantenna that undergoes efficient Förster Resonance Energy Transfer (FRET) from 10 000 donor dyes to very few acceptors inside the nanoparticle, yielding an amplification of the acceptor signal of 1000 times (Trofymchuk et al., 2017). These nanoantennes were then functionalized with DNA yielding nanoprobes for nucleic acids, stable in physiological conditions and operating by FRET with strand displacement mechanism (Melnychuk and Klymchenko, 2018). The obtained nanoprobes, being 100 times brighter than QDot-605, produced a donor/acceptor ratio response to single hybridization events (Melnychuk et al., 2020).

In a single-molecule FRET detection, highly sensitive monochromatic cameras are used with an image splitting module (Roy et al., 2008), which is a highly costly and difficult to handle considering point of care devices. Modern consumer grade cameras are comprised of a color filter array for red, green and blue light (RGB) overlaid on an active pixel sensor array based on a complementary metal-oxide semiconductor (CMOS) light sensor (Takayanagi, 2006). These cameras allow the simultaneous detection of three spectral channels and can provide a rapid and simple detection of color change in FRET nanoprobes (Wang et al., 2011). FRET detection assays with the RGB camera of a smartphone is an emerging field (Noor and Krull, 2014; Petryayeva and Algar, 2015; Yan et al., 2018). However, smartphone-based FRET detection at the single nanoparticle level has not been achieved so far, which would open the opportunity to ultrasensitive point-of-care detection. Light-harvesting dye-loaded polymeric NPs, 
owing to their exceptional brightness, offer this opportunity; but in these nanoprobes both donor and acceptor emit in the red region (Melnychuk et al., 2020; Melnychuk and Klymchenko, 2018), which make these probes not compatible with common RGB cameras.

In this work, we redesigned these light-harvesting nanoprobes for nucleic acids to make them compatible with RGB detection. We selected a donor-acceptor FRET pairs with emissions compatible with the red and green channels of the RGB camera and designed nanoprobe for a nucleic acid fragment encoding survivin, an important anti-apoptotic cancer marker (Altieri, 2003; Stobiecka et al., 2019). We found that the optimized nanoprobe provides a ratiometric response to the target nucleic acid with limit of detection in solution of $3 \mathrm{pM}$. Remarkably, our nanoprobes, immobilized on glass surface, enabled detection of nucleic acids at the single-particle level, using RGB microscopy camera as well as a smartphone camera. We show that our biosensor system can work with an inexpensive consumer-grade RGB camera, using low excitation power densities. These results open the route to very simple smartphone-based biosensing devices for point-of-care detection of nucleic acid markers of diseases.

\section{Results and discussions}

\subsection{Design of RGB compatible FRET-based nanoprobes}

The design of the nanoparticle probe is based on DNA-functionalized nanoantenna, which serves as a FRET donor for a FRET acceptor conjugated to a short oligonucleotide hybridized at the particles surface (Fig. 1A,B). Then, hybridization with the target nucleotide displaces the acceptor leading to FRET turn off and thus a color switch (Melnychuk and Klymchenko, 2018; Melnychuk et al., 2020). To obtain a system compatible with RGB detection, it is necessary to select a FRET pair where emission of the donor and the acceptor is compatible with two distinct RGB channels with minimal crosstalk (Fig. 1C,D). To this end, we designed as the FRET donors the green-emitting octadecyl ester of Rhodamine 110 (R110-C18) and the yellow-emitting octadecyl ester of Rhodamine 6G (R6GC18). Both of them emit in the green channel region of the RGB systems (Figure 1D and 2A). They were synthesized by esterification of corresponding acid form of the dyes with octadecanol in acidic conditions. ATTO 647N (Fig. 3B) was chosen as the FRET acceptor because its absorption overlaps well with the emission of the donor dyes, thus ensuring an efficient FRET, and its emission is compatible with the RGB red channel (Figure 1D).
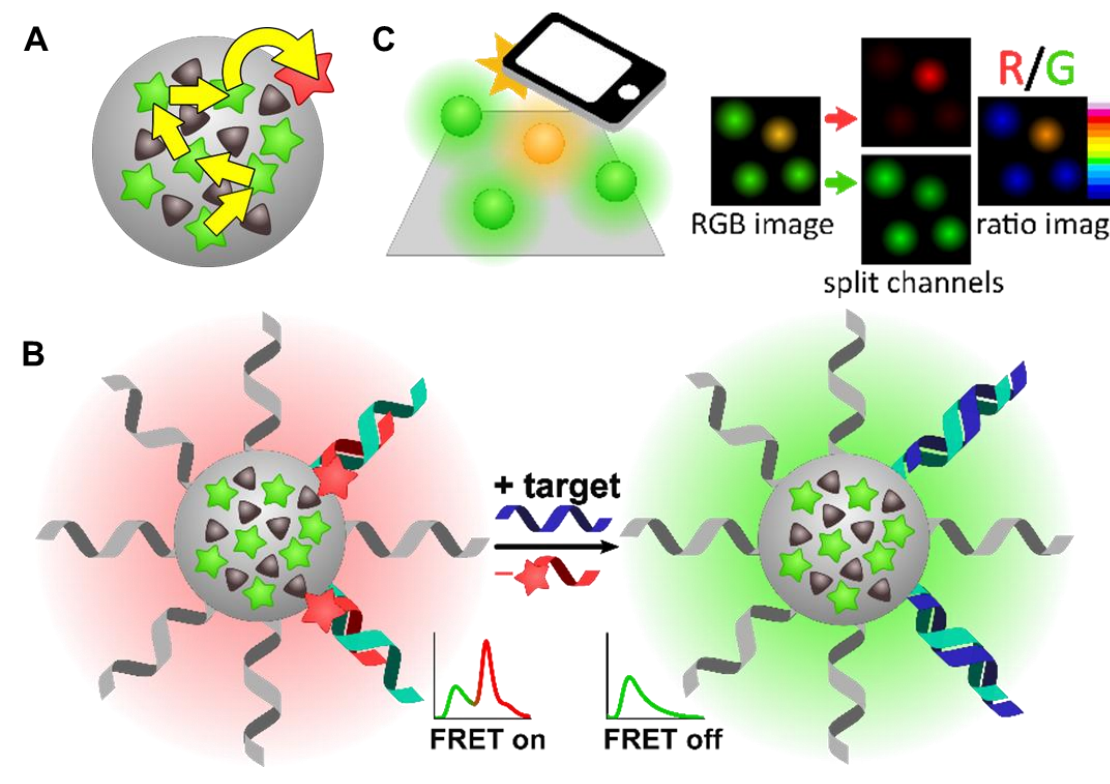

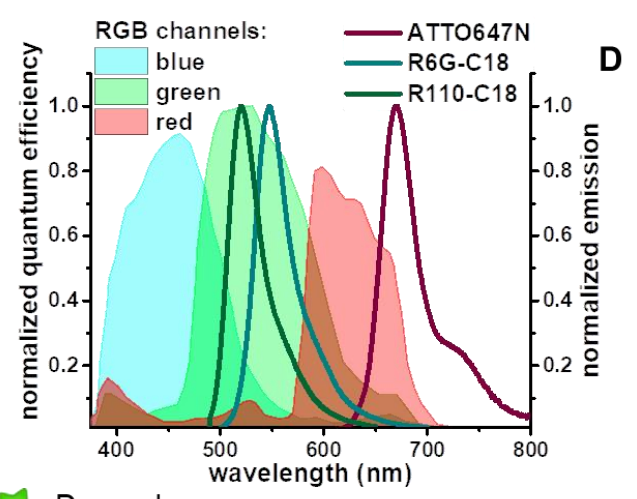

Donor dye

Counterion

Acceptor dye

Capture sequence (SurC)

Noncoding sequence (T20)

Acceptor-target competitive sequence (TCS) 
Fig. 1. DNA nanoprobes for smartphone-based RGB detection of nucleic acids. (A) Light-harvesting in the nanoantenna particles: donor-donor excitation energy transfer inside NPs is followed by FRET to the acceptor at the NP surface. (B) Nanoprobe design: the green donors inside the NP transfer part of their excitation energy towards FRET acceptor at the surface, making the fluorescence from the nanoprobe yellow-orange. In presence of the target sequence the sequence bearing the FRET acceptor is displaced, turning off FRET and switching the emission color to green. (C) Smartphone-based data analysis workflow: an RGB image is taken and the ratio between the red and green channel is evaluated; this ratio is a function of target concentration. (D) Comparison between the spectral response (quantum efficiency) of the RGB channels of the color camera (Nikon DS-Fi3) with the emission spectra in ethanol of the dyes selected for the nanoprobe design.

\subsection{Donor NPs preparation}

Polymeric NPs were prepared based on a polymer derivative of poly(methyl methacrylate-co-methacrylic acid (PMMA-MA, 1.6\% methacrylic acid) (Fig. 2A) bearing azide groups (PMMA-AspN3) as was previously described (Melnychuk and Klymchenko, 2018) and loaded with donor dye paired with a bulky hydrophobic counterion. The role of the counterion is to insulate the dye molecules in order to prevent their aggregation-caused quenching (ACQ) (Reisch et al., 2014) and to ensure their efficient encapsulation without dye leakage in biological environment (Andreiuk et al., 2017b). Moreover, the presence of counterion creates a supramolecular organization of dyes and counterions which produce an ultrafast excitation energy migration allow efficient FRET from thousands of donor dyes to few acceptors (Trofymchuk et al., 2017). We chose to test two different counterions: one based on a fluorinated tetraphenylborate (F12) and tetrakis(perfluoro-tert-butoxy)aluminate (F9-Al) (Krossing, 2001), because of their already established effectiveness (Andreiuk et al., 2017a, 2017b) (Fig. 2A).

The two donor dyes were paired with the two counterions, using ion exchange method (Reisch et al., 2014), for a total of four ion pairs. Then, NPs were prepared by nanoprecipitation of the polymer with these four pairs at three different loadings $(10,120$ and $250 \mathrm{mM}$ with respect to the polymer). Charged carboxylate group in the polymer ensures controlled formation of the small particles during nanoprecipitation due to electrostatic repulsion (Reisch et al., 2018, 2017). The as-prepared samples were characterized by dynamic light scattering (DLS) and optical spectroscopy. Hydrodynamic diameters of NPs remained relatively stable, in the range of 35-50 nm, independently of dye loading and ion pair (Table S1 of the Supplementary Materials). The increase in the dye loading resulted in some broadening of the absorption and emission spectra, indicating the effect of dye aggregation in the polymer matrix. These changes were accompanied by blue shifts in the absorption spectra and red shifts in the emission spectra, which were more pronounced for the R6G-C18 dyes (Figure S1 of the Supplementary Materials). For all ion pairs the fluorescence quantum yields (QY) decreased with the loading due to some ACQ. Nevertheless, R6GC18/F12 loaded NPs displayed relatively high QY (Fig. 2C), around 20\% at $250 \mathrm{mM}$ dye loading (i.e. $~ 6000$ dyes per particle) and the emission band for all dye salts remained relatively narrow (Fig. 2D). 

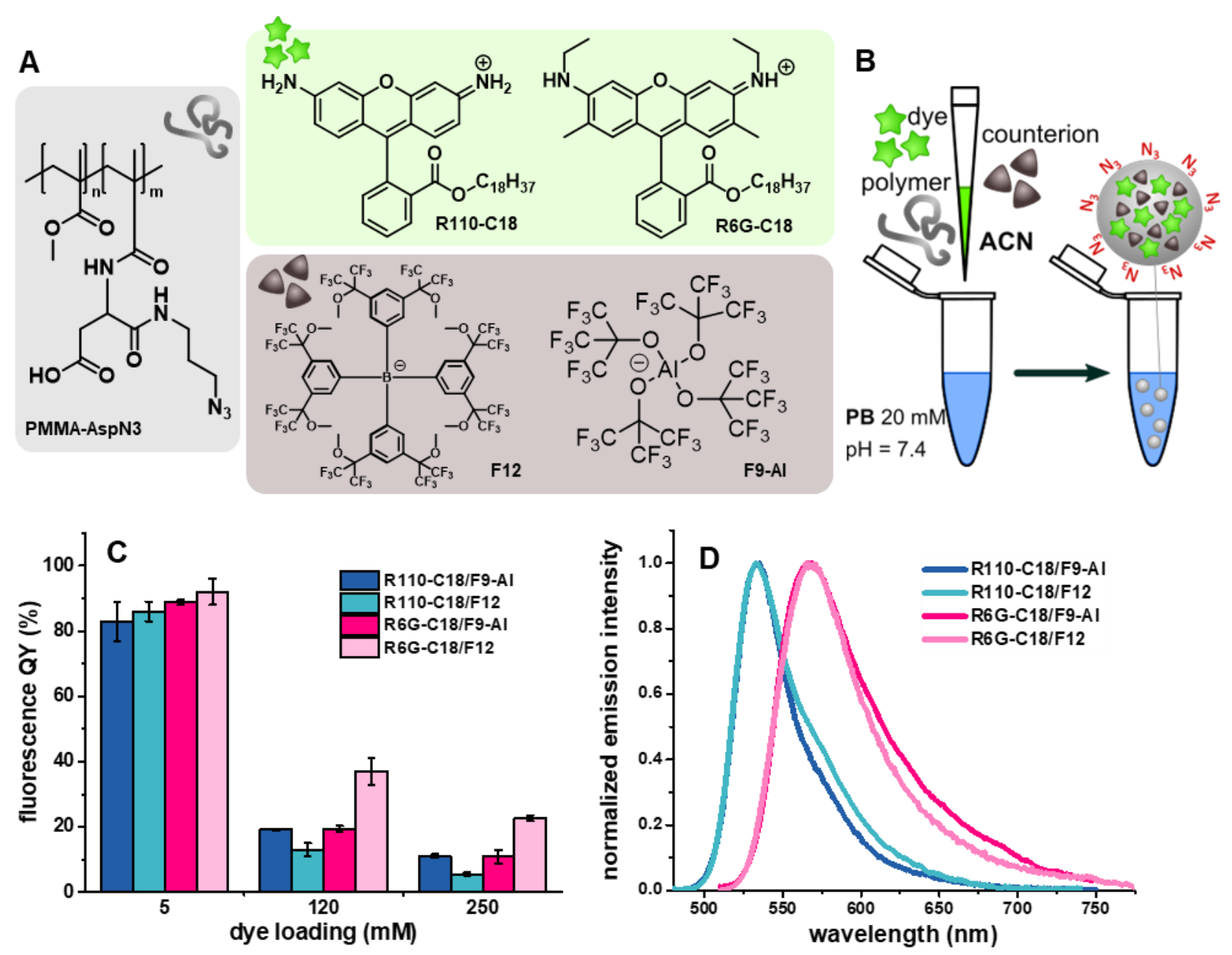

Fig. 2. Design and fluorescence properties of light-harvesting nanoantenna particles (donor NPs). (A) Azidemodified polymer PMMA-AspN3 (left), dyes (upper row) and counterions (lower row) used for the nanoparticles preparation. (B) Nanoparticles preparation scheme: nanoprecipitation from acetonitrile (ACN) to phosphate buffer (PB). (C) Quantum yields of NPs prepared with different dye salts at different loadings. Error bars are standard deviation $(\mathrm{n}=3)$. (D) Fluorescence spectra for the NPs formulated with the four different dye salts at $250 \mathrm{mM}$ loading.

\subsection{FRET-based DNA nanoprobes preparation}

Ion pair R110-C18/F12 was excluded from FRET studies, because of its too low quantum yield (Fig. 2C). For the remaining ion pairs, NPs loaded with $250 \mathrm{mM}$ and different quantities of FRET acceptor (DiD, Figure S3, Supplementary Material) were prepared by co-precipitating donor and acceptor dyes together and their FRET properties were studied (Figures S4-S6, Supplementary Material). It was found that both R6G-C18/F12 and R6GC18/F9-Al performed better in terms of FRET efficiency than R110-C18/F9-Al (Figure S7, Supplementary Material). Moreover, antenna effect (AE), which is a value indicating the magnitude of acceptor amplification by the nanoantenna particle measured by the excitation spectrum (Trofymchuk et al., 2017; Woller et al., 2013), was much higher R6G-C18/F12 and R6G-C18/F9-Al NPs compared to R110-C18/F9-Al NPs. Therefore, we selected R6G-C18 salts for fabrication of FRET-based DNA nanoprobes, bearing the hybridized FRET acceptor (TCSATTO647N, which has similar spectral properties to DiD) at the NP surface. After nanoprecipitation (Fig. 2B) azide-bearing NPs were functionalized by clicking oligonucleotide sequences bearing dibenzocyclooctyne (DBCO) reactive group, and subsequently annealed with an acceptor-bearing complimentary sequence (TCS-ATTO647N) 
(Fig. 3A), following a previously described methodology (Melnychuk and Klymchenko, 2018). To control the amount of capture sequence (SurC) on NPs surface, SurC-DBCO was added to NPs at different concentrations $(0.5$ $\mu \mathrm{M}, 1.5 \mu \mathrm{M}$ and $3 \mu \mathrm{M}$ ) together with a non-coding DNA-DBCO (T20), while keeping constant the total amount of added DNA-DBCO $(23 \mu \mathrm{M})$. Then, the obtained DNA-functionalized NPs were hybridized with TCSATTO647N and purified by ultrafiltration. The amount of hybridized TCS-ATTO647N on the surface of the nanoparticles was quantified by absorption spectroscopy (Fig. S8 of the Supplementary Materials). For each SurC concentration the number of TCS-ATTO647N acceptors per NP was, respectively, 5, 30 and 65 acceptors per particle.

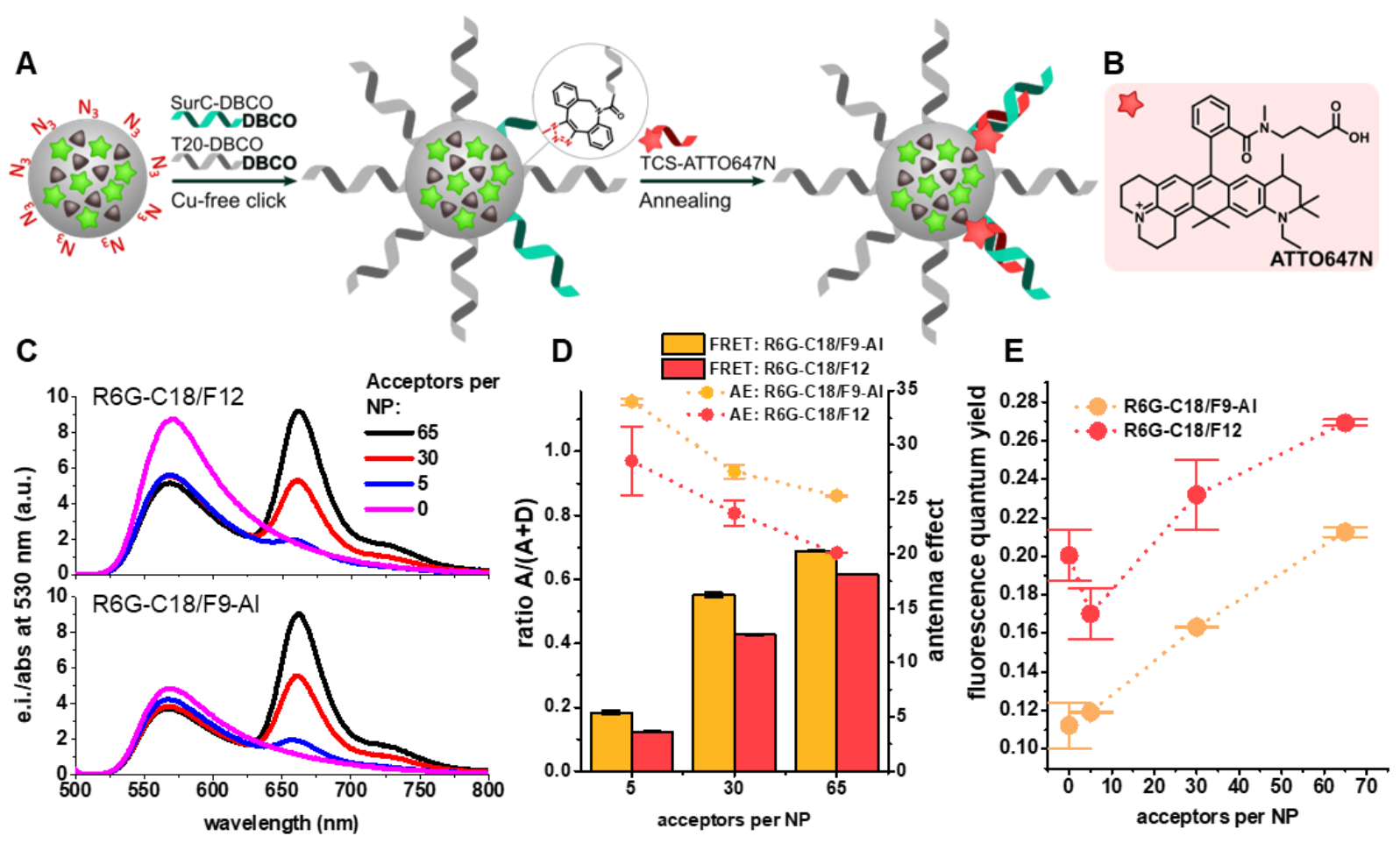

Fig. 3. Preparation, optical properties and antenna effect of DNA nanoprobes. (A) Preparation of nanoprobe: functionalization of dye-loaded nanoparticles with DNA and subsequent annealing with the FRET acceptor bearing oligonucleotide. (B) Structure of the FRET acceptor ATTO647N. (C) Fluorescence spectra, (D) FRET ratio (semiquantitative FRET efficiency), antenna effect, and (E) quantum yield of NPs loaded at $250 \mathrm{mM}$ of R6G-C18 with two different counterions (F12 and F9-Al) with increasing number of ATTO647N acceptors per NP. Error bars are standard deviation $(\mathrm{n}=3)$.

For both tested donor dye salts, the increase in the number of acceptors per NP increased relative intensity of the acceptor emission, accompanied by a small decrease in the donor emission (Figure 3C and S9A, Supplementary Material). These spectral changes indicate that FRET takes place from donor NPs to ATTO647N acceptor at the surface. FRET efficiency, expressed as the empirical FRET ratio $\mathrm{A} /(\mathrm{A}+\mathrm{D})$, increased with the increase of the number of acceptors. The FRET ratio was slightly higher for R6G-C18/F9-Al (Figure 3D). The total QY value of donor and acceptor also increased in the presence of acceptor, especially in the case R6G-C18/F9-Al NPs, where QY increased from $0.11 \pm 0.01$ for NPs without acceptor, to $0.212 \pm 0.002$ for those with 65 acceptors per particle (Fig. 3E). This QY enhancement could be due to the fact that a part of the excitation energy transferred to the acceptor derives from the non-emissive self-quenched donor dyes, thus producing a de-quenching effect on the whole system, as shown for other NPs (Genovese et al., 2013). For both donor dye salts (R6G-C18/F12 and R6GC18/F9-Al) the antenna effect increased with decrease in the number of acceptors per NP. R6G-C18/F9-Al NPs performed slightly better than R6G-C18/F12 NPs, with its AE ranging from 25 till 34 for number of acceptors per 
NP varied from 65 till 5. The latter means that the signal of the acceptor is amplified 25-34 times due to the lightharvesting effect of the nanoantenna, where a large number of donor dyes inside nanoparticle ( 6000 per 43-nm particle) undergo efficient FRET to a few acceptors at the surface. R6G-C18/F9-Al NPs were selected for the experiments on the DNA target detection. Taking into account the number of encapsulated dyes (n), extinction coefficient of Rhodamine $6 \mathrm{G}\left(116,000 \mathrm{M}^{-1} \mathrm{~cm}^{-1}\right)$ and their quantum yield (QY), we can estimate the brightness of R6G-C18/F9-Al nanoprobe: $\mathrm{B}=\mathrm{n} \times \varepsilon \times \mathrm{QY}=6000 \times 116,000 \mathrm{~cm}^{-1} \mathrm{M}^{-1} \times 0.212=1.48 \times 10^{8} \mathrm{M}^{-1} \mathrm{~cm}^{-1}$. This value is equivalent of $\sim 2000$ high-performance dyes with typical extinction coefficient of $150,000 \mathrm{M}^{-1} \mathrm{~cm}^{-1}$ and QY of $0.5\left(\mathrm{~B}=7.5 \times 10^{4} \mathrm{M}^{-1} \mathrm{~cm}^{-1}\right)$ and of $\sim 135$ Qdots-605 $\left(\mathrm{B}=1.1 \times 10^{6} \mathrm{M}^{-1} \mathrm{~cm}^{-1}\right.$, excitation at $488 \mathrm{~nm}$, assuming QY = $1)$.
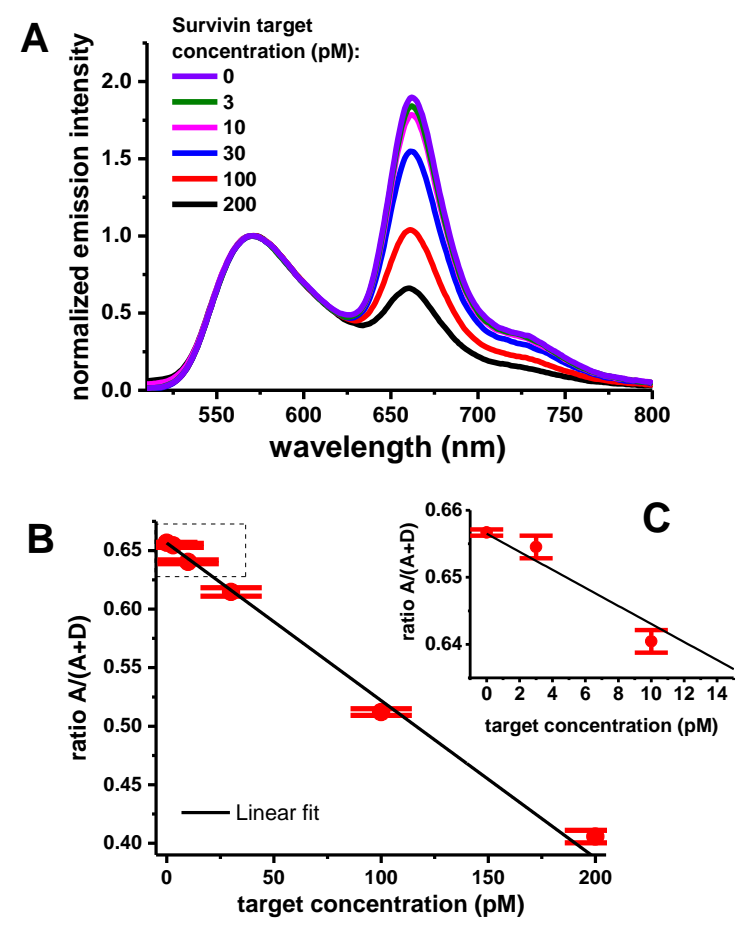

Fig. 4. Ratiometric detection of the DNA target. (A) Fluorescence spectra of NP-probe-65 incubated with increasing concentrations of the DNA target sequence. The spectra were normalized at the donor intensity. (B) FRET ratio as a function of target concentration. (C) Data points in the square shown in (B). Error bars are standard deviation (n $=3$ ). 


\subsection{Detection of target DNA sequence}

All the following experiments were performed on the R6G-C18/F9-Al-loaded NP-probe with 65 acceptors per NP (NP-probe-65). In the nanoprobe design, DNA target sequence, encoding survivin fragment (20mer ssDNA), displaces the TCS-ATTO647N acceptor from NP surface, thus turning off the FRET signal (Fig. 1B). Indeed, after incubation with the target, the signal corresponding to the acceptor at $662 \mathrm{~nm}$ decreased. This confirmed that the FRET acceptor was hybridized at the NP surface instead of just being nonspecifically absorbed, so that the target effectively displaced the TCS-ATTO647N and turned off the FRET. This result is in line with our previous FRETbased probes operating in the red region (Melnychuk et al., 2020; Melnychuk and Klymchenko, 2018). To optimize sensitivity of our assay, we diluted the NPs suspension to $10 \mathrm{pM}$ of the acceptor (corresponding to $\sim 0.15 \mathrm{pM}$ of NPs) and incubated it (6 h, rt) with the DNA target at different concentrations (3-200 pM). We observed that, with increase in the target concentration, the acceptor relative intensity decreased gradually with some recovery of donor fluorescence (Figure S11), showing almost linear change of the FRET ratio vs target concentration (Fig. 4). The limit of detection and the limit of quantitation (LOD and LOQ, defined as, respectively, 3 and 10 times the ratio between the standard error of the control without target and the slope of the linear regression (Analytical Methods Committee, 1987) (see Table S2, Supplementary Material) for this assay were 3 and 9 pM, respectively.

To study the response of NP-probe-65 at the single-particle level, it was immobilized on the glass surface using a sandwich of biotinylated BSA, neutravidin and biotinylated A20, followed by a hybridization of the nanoprobe (Fig. 5A) (Melnychuk and Klymchenko, 2018). Immobilized NP-probe-65 was visualized via fluorescence microscopy using both a sCMOS (Hamamatsu Orca Flash 4.0) camera equipped with a beam splitter (W-VIEW GEMINI) and an RGB camera (Nikon, DS-Fi3). The beam splitter at $640 \mathrm{~nm}$ allowed us to separately detect donor an acceptor channels and then to construct merged and ratio images. The increase in the target concentration decreased gradually the intensity in the acceptor channel, so that on the merged images the particles turned from orange to green (Fig. 5B). In the ratio images drastic change of NPs color towards blue (low acceptor/donor ratio) was observed, in line with the spectroscopic data in solution (Fig. 4). A histogram of the ratio distribution shows that ratio of the NP-probe-65 initially centered at $\sim 0.6$ shifted gradually to $\sim 0.4$ and $\sim 0.2$ for 10 and $1000 \mathrm{pM}$ of the target, respectively (Fig. 5D), indicating that this method could in principle allow quantification of the target. On the other hand, for RGB color camera, merged images shoed only small color change from orange to yellow with addition of the $1000 \mathrm{pM}$ DNA target. The differences in performance between the sCMOS camera and the RGB camera could be due to two reasons: (i) sCMOS has an higher sensitivity and dynamic range to the RGB sensor and (ii) the use of the beam splitter with a dichroic at $640 \mathrm{~nm}$ provides efficient separation of donor and acceptor signals of NP-probe-65 than green and red channels of the RGB sensor. Nevertheless, the ratio images for the RGB camera (Fig. 5C) showed clear changes in the red/green ratio in response to the target (color change from orange to blue), which was confirmed by the ratio histogram (Fig. 5D). 

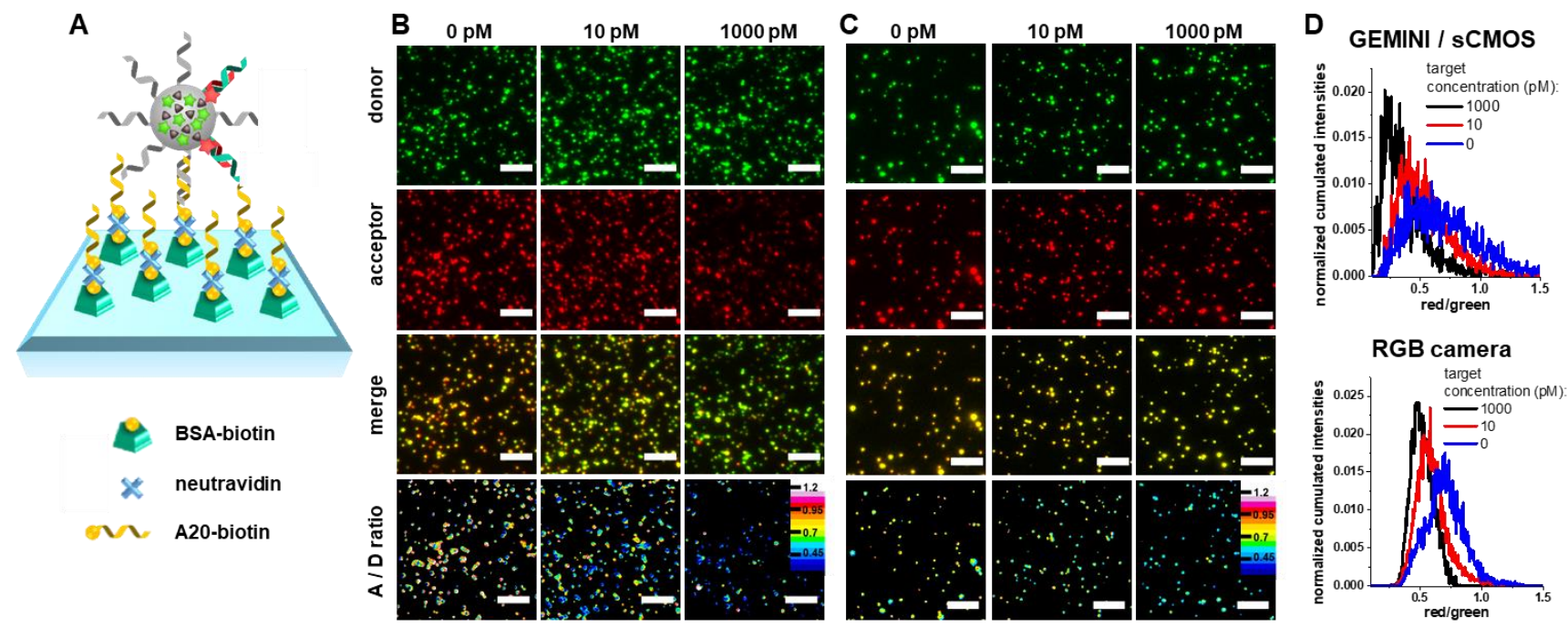

Fig. 5. Nucleic acid detection by NP-probe-65 on glass surfaces using scientific-grade sCMOS with image splitting (GEMINI) and RGB camera. (A) Surface immobilization of nanoprobe for microscopy experiments. (B) and (C): Detection of DNA target (survivin) at the single-particle level using sCMOS (Hamamatsu Orca Flash 4) with image splitter (B) and RGB (Nikon DS-Fi3) camera (C). Samples were incubated for $3 \mathrm{~h}$ (rt) with nucleic acid target at different concentrations. From up to down, fluorescence microscopy images: donor channel, acceptor channel, donor + acceptor merged image, and ratiometric image of the acceptor (red) over the donor (green) channel. Excitation was at $488 \mathrm{~nm}$ with irradiance of $2 \mathrm{~W} \mathrm{~cm}^{-2}$. (D) Ratio distribution histograms for the corresponding ratiometric images using the two camera types. Scale bars: $5 \mu \mathrm{m}$.

Finally, we challenged our NP-probe-65 biosensor by imaging it on glass surface using a cellphone camera (iPhone SE, Backlit Sony Exmor RS 12 MP, $1.22 \mu \mathrm{m}, \mathrm{f} / 2.2$ aperture). The detection setup was simply constructed by replacing the Nikon RGB camera with a smartphone together with an eyepiece (10x) at the exit of the microscope (Fig. 6A). The estimated pixel size in our images was $0.526 \mu \mathrm{m} / \mathrm{px}$ (see Supplementary Materials 1.3.8). Using a special smartphone application that allows controlling key camera parameters (ISO, exposure time, aperture), we acquired images where individual particles could be readily identified. Remarkably, the true-color images recorded with a smartphone could directly show the change in the color of NP-probe- 65 in the presence of $10 \mathrm{pM}$ target. The color change from orange to yellow-green corresponded to loss of FRET in NP-probe-65, expected for the strand displacement induced by the DNA target (Fig. 6B). Red/green channel ratio images revealed drastic changes in the ratio from red pseudo-color to green-yellow, green-blue and blue for 10, 100 and 1000 pM target, respectively (Fig. 6C). The ratio histograms confirmed the gradual change in the red/green ratio, and distribution histograms looked better separated compared to Nikon RGB camera (Fig. 6D vs 5D). The major response was observed already at 10 pM target, probably because at this concentration corresponded to $\sim 10$-fold access with respect to the FRET acceptor (TCS-ATTO647N). Thus, the ratiometric response of FRET-based nanoprobe to target DNA can be readily recorded with a smartphone camera using a standard epi-fluorescence optical microscope. 


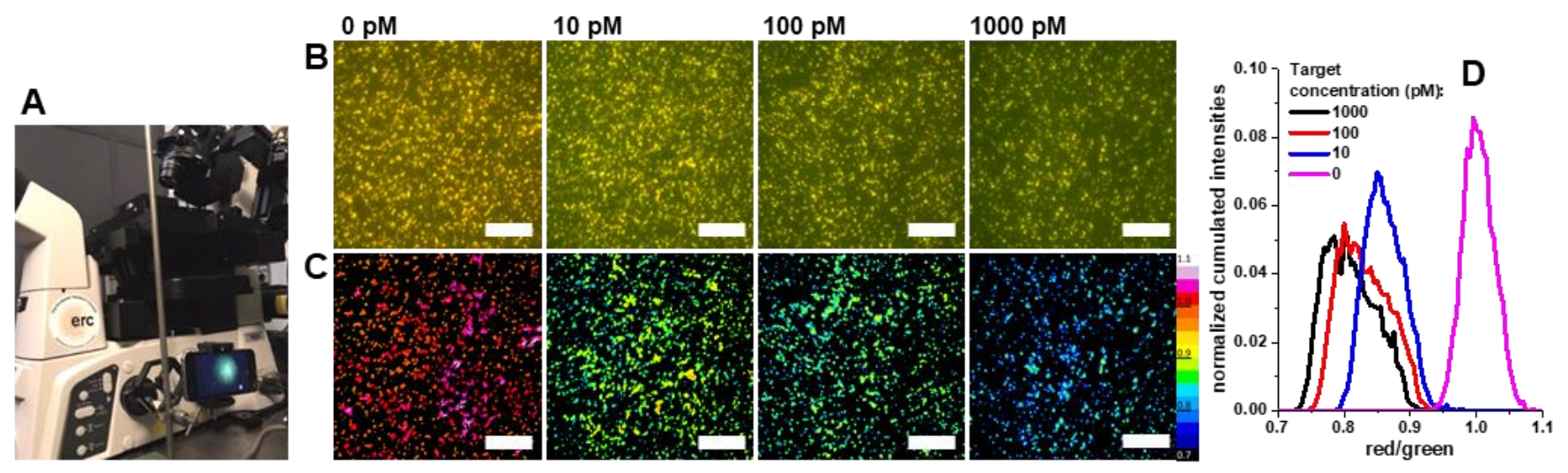

Fig. 6. Smartphone-based detection of nucleic acids by NP-probe-65 on glass surface. (A) Smartphone-based detection setup. (B) RGB true-color images acquired with a smartphone (iPhone SE). Images were acquired after $3 \mathrm{~h}$ of incubation (rt) with DNA target (survivin) at different concentrations. (C) Ratiometric images of the red over the green channel from the images shown in (B). Excitation was at $488 \mathrm{~nm}$ with irradiance of $2 \mathrm{~W} \mathrm{~cm}^{-2}$. (D) Ratio distribution histograms for the images shown in (C). Scale bars: $25 \mu \mathrm{m}$.

\section{Conclusion}

In this work, a FRET-based DNA nanoprobe was developed for detection with a smartphone camera. To this end, we designed 40-nm PMMA-based polymeric NPs encapsulating green emitting fluorescent dyes, octadecyl Rhodamines 110 and 6G, paired with bulky counterions for preventing aggregation-caused quenching in the polymer matrix. Their surface was functionalized with DNA complementary to the sequence encoding cancer marker survivin, which is hybridized with the target competitive sequence bearing red emitting FRET acceptor ATTO647N. Among tested dye salts, octadecyl Rhodamine 6G with aluminum-based bulky counterion, showed best FRET characteristics, while the optimal acceptor concentration was 65 molecules per NP. The obtained nanoprobe displayed fluorescence quantum yield $>20 \%$ and antenna effect of 25 . The nanoprobe showed ratiometric response to the target DNA in solution with a limit of detection of $3 \mathrm{pM}$. Single-particle fluorescence microscopy of immobilized nanoprobe revealed that both sCMOS camera with a beam splitter and RGB camera can detect the color switch of the FRET nanoprobe in presence of varying concentrations of the DNA target. Finally, our nanoprobe enabled detection of 10-pM DNA target using RGB camera of a smartphone in true color or ratio (red-to-green) imaging at the single-particle level. Thus, high brightness of NPs and signal amplification through light harvesting allowed DNA sensing by a smartphone sensor coupled to an appropriate optical setup. We foresee that the optical part could be greatly simplified, which would open the way to simple and inexpensive smartphonebased point-of-care assays for detection of biomolecular markers of diseases.

\section{Declaration of competing interest}

Nina Melnychuk and Andrey S. Klymchenko are co-inventors of a patent application: European patent application $\mathrm{n}^{\circ}$ 18305253.9. The authors declare that they have no other known competing financial interests or personal relationships that could have appeared to influence the work reported in this paper.

\section{Authorship Contribution statement}


Caterina Severi: most of the experimental work, data analysis, paper writing. Nina Melnychuk: synthesis of the polymer and some dyes, help with nanoprobe synthesis, manuscript reviewing. Andrey S. Klymchenko: conceptualization, supervision of the project, help with data analysis and fluorescence microscopy, paper writing.

\section{Acknowledgments}

This work, CS and NM were supported from European Research Council ERC Consolidator grant BrightSens 648528 and SATT Conectus Alsace grant Nanoantenna. We thank I. Krossing for providing sample of lithium tetrakis(perfluoro-tert-butoxy)aluminate.

\section{Appendix A. Supplementary data}

Supplementary data to this article can be found online at https://doi.org

\section{References}

Acuna, G.P., Möller, F.M., Holzmeister, P., Beater, S., Lalkens, B., Tinnefeld, P., 2012. Science 338, $506-510$.

Ali, M.M., Li, F., Zhang, Z., Zhang, K., Kang, D.-K., Ankrum, J.A., Le, X.C., Zhao, W., 2014. Chem. Soc. Rev. 43, 3324.

Altieri, D.C., 2003. Oncogene 22, 8581-8589.

Analytical Methods Committee, 1987. The Analyst 112, 199.

Andreiuk, B., Reisch, A., Bernhardt, E., Klymchenko, A.S., 2019. Chem. - Asian J. 14, 836-846.

Andreiuk, B., Reisch, A., Lindecker, M., Follain, G., Peyriéras, N., Goetz, J.G., Klymchenko, A.S., 2017 a. Small 13, 1701582.

Andreiuk, B., Reisch, A., Pivovarenko, V.G., Klymchenko, A.S., 2017b. Mater. Chem. Front. 1, 23092316.

Breslauer, D.N., Maamari, R.N., Switz, N.A., Lam, W.A., Fletcher, D.A., 2009. PLOS ONE 4, e6320.

Chen, A., Chatterjee, S., 2013. Chem. Soc. Rev. 42, 5425.

Choi, H.M.T., Chang, J.Y., Trinh, L.A., Padilla, J.E., Fraser, S.E., Pierce, N.A., 2010. Nat. Biotechnol. 28, 1208-1212.

Coskun, A.F., Nagi, R., Sadeghi, K., Phillips, S., Ozcan, A., 2013. Lab. Chip 13, 4231-4238.

Dakshayini, B.S., Reddy, K.R., Mishra, A., Shetti, N.P., Malode, S.J., Basu, S., Naveen, S., Raghu, A.V., 2019. Microchem. J. 147, 7-24.

Genovese, D., Bonacchi, S., Juris, R., Montalti, M., Prodi, L., Rampazzo, E., Zaccheroni, N., 2013. Angew. Chem. Int. Ed. 52, 5965-5968.

Grimm, J.B., English, B.P., Chen, J., Slaughter, J.P., Zhang, Z., Revyakin, A., Patel, R., Macklin, J.J., Normanno, D., Singer, R.H., Lionnet, T., Lavis, L.D., 2015. Nat. Methods 12, 244-250.

Jiang, Y., McNeill, J., 2017. Chem. Rev. 117, 838-859.

Kanchi, S., Sabela, M.I., Mdluli, P.S., Inamuddin, Bisetty, K., 2018. Biosens. Bioelectron. 102, $136-149$. Krossing, I., 2001, Chem. - Eur. J., 7, 490-502.

Kühnemund, M., Wei, Q., Darai, E., Wang, Y., Hernández-Neuta, I., Yang, Z., Tseng, D., Ahlford, A., Mathot, L., Sjöblom, T., Ozcan, A., Nilsson, M., 2017. Nat. Commun. 8.

Melnychuk, N., Egloff, S., Runser, A., Reisch, A., Klymchenko, A.S., 2020. Angew. Chem. Int. Ed.

Melnychuk, N., Klymchenko, A.S., 2018. J. Am. Chem. Soc. 140, 10856-10865.

Noor, M.O., Krull, U.J., 2014. Anal. Chem. 86, 10331-10339.

Petryayeva, E., Algar, W.R., 2015. The Analyst 140, 4037-4045. 
Reisch, A., Didier, P., Richert, L., Oncul, S., Arntz, Y., Mély, Y., Klymchenko, A.S., 2014. Nat. Commun. $5,4089$.

Reisch, A., Heimburger, D., Ernst, P., Runser, A., Didier, P., Dujardin, D., Klymchenko, A.S., 2018. Adv. Funct. Mater. 28, 1805157.

Reisch, A., Klymchenko, A.S., 2016. Small 12, 1968-1992.

Reisch, A., Trofymchuk, K., Runser, A., Fleith, G., Rawiso, M., Klymchenko, A.S., 2017. ACS Appl. Mater. Interfaces 9, 43030-43042.

Rochat, S., Swager, T.M., 2013. ACS Appl. Mater. Interfaces 5, 4488-4502.

Roy, R., Hohng, S., Ha, T., 2008. Nat. Methods 5, 507-516.

Roy, S., Malode, S.J., Shetti, N.P., Chandra, P., 2019, Modernization of Biosensing Strategies for the

Development of Lab-on-Chip Integrated Systems, in: Krishnaraj, R.N., Sani, R.K.. Bioelectrochemical Interface Engineering. John Wiley \& Sons, Hoboken, NJ, pp 325-342.

Scrimin, P., Prins, L.J., 2011. Chem. Soc. Rev. 40, 4488-4505.

Shetti, N.P., Bukkitgar, S.D., Reddy, K.R., Reddy, Ch.V., Aminabhavi, T.M., 2019. Biosens. Bioelectron. $141,111417$.

Stobiecka, M., Ratajczak, K., Jakiela, S., 2019. Biosens. Bioelectron. 137, 58-71.

Takayanagi, I., 2006, CMOS Image Sensor, in: Nakamura, J. (Ed.), 2006. Image sensors and signal processing for digital still cameras. Taylor \& Francis, Boca Raton, FL, pp 143-178.

Trofymchuk, K., Reisch, A., Didier, P., Fras, F., Gilliot, P., Mely, Y., Klymchenko, A.S., 2017. Nat. Photonics 11, 657-663.

Wang, X., Gorris, H.H., Stolwijk, J.A., Meier, R.J., Groegel, D.B.M., Wegener, J., Wolfbeis, O.S., 2011. Chem. Sci. 2, 901.

Wei, Q., Acuna, G., Kim, S., Vietz, C., Tseng, D., Chae, J., Shir, D., Luo, W., Tinnefeld, P., Ozcan, A., 2017. Sci. Rep. 7, 1-10.

Wei, Q., Luo, W., Chiang, S., Kappel, T., Mejia, C., Tseng, D., Chan, R.Y.L., Yan, E., Qi, H., Shabbir, F., Ozkan, H., Feng, S., Ozcan, A., 2014. ACS Nano 8, 12725-12733.

Wei, Q., Qi, H., Luo, W., Tseng, D., Ki, S.J., Wan, Z., Göröcs, Z., Bentolila, L.A., Wu, T.-T., Sun, R., Ozcan, A., 2013. ACS Nano 7, 9147-9155.

Woller, J.G., Hannestad, J.K., Albinsson, B., 2013. J. Am. Chem. Soc. 135, 2759-2768.

Xu, H., Xia, A., Wang, D., Zhang, Y., Deng, S., Lu, W., Luo, J., Zhong, Q., Zhang, F., Zhou, L., Zhang, W., Wang, Y., Yang, C., Chang, K., Fu, W., Cui, J., Gan, M., Luo, D., Chen, M., 2020. Sci. Adv. 6, eaaz7445.

Yan, Q., Chen, Z.-H., Xue, S.-F., Han, X.-Y., Lin, Z.-Y., Zhang, S., Shi, G., Zhang, M., 2018. Sens. Actuators B Chem. 268, 108-114.

Yang, M., Liu, Y., Jiang, X., 2019. Chem. Soc. Rev. 48, 850-884.

Zhang, D., Liu, Q., 2016. Biosens. Bioelectron. 75, 273-284.

Zhu, H., Sikora, U., Ozcan, A., 2012. The Analyst 137, 2541-2544. 